\title{
Enhanced diffusion and bound exciton interactions of high density implanted bismuth donors in silicon
}

\author{
T. Peach, ${ }^{1}$ K. Stockbridge, ${ }^{1}$ Juerong. Li, ${ }^{1}$ K. P. Homewood, ${ }^{2,3}$ M. A. Lourenco, ${ }^{2,3}$ S. Chick, ${ }^{1}$ M. A. Hughes, ${ }^{1,4}$ \\ B. N. Murdin, ${ }^{1}$ and S. K. Clowes ${ }^{1, \text { a) }}$ \\ 1) Department of Physics, University of Surrey, Guildford, Surrey, GU2 7XH UK \\ ${ }^{2)}$ Department of Electrical and Electronic Engineering, University of Surrey, Guildford, Surrey, \\ GU2 $7 X H U K$ \\ ${ }^{3)}$ School of Materials Science \& Engineering, Hubei University, Wuhan 430062 PR China \\ 4) Joule Physics Laboratory, School of Computing Science and Engineering, University of Salford, \\ Salford M5 4WT UK
}

(Dated: 18 July 2019)

\begin{abstract}
This study reports the effect of an increasing ion dose on both the electrical activation yield and characteristic properties of implanted bismuth donors in silicon. A strong dependence of implant fluence is observed on both the yield of bismuth donors and the measured impurity diffusion. This is such that higher ion concentrations result in both a decrease in activation and an enhancement in donor migration through interactions with mobile silicon lattice vacancies and interstitials. Furthermore, the effect of implant fluence on the properties of the $\mathrm{Si} B \mathrm{Bi}$ donor bound exciton, $D^{0} X$, is also explored using photoluminescence, PL, measurements. In the highest density sample, centers corresponding to the PL of bismuth $D^{0} X$ s within both the high density region and the lower concentration diffused tail of the implanted donor profile are identifiable.
\end{abstract}

Bismuth donors in silicon are promising candidates for implementation as spin qubits due to their extremely long electron and nuclear spin coherence times ${ }^{1}$ and strong hyperfine interaction $^{2,3}$. This promise has lead to a great deal of research into the implementation of bismuth donors as qubit states in recent years. Such interest has included studies into their incredibly robust so called clock transitions ${ }^{4}$ and utilising the large donor ground state hyperfine splitting for the optical hyperpolarisation of bismuth donors in the absence of a magnetic field ${ }^{5}$. Fabrication of bismuth donors in silicon on a device scale is now required and the leading mechanism of incorporation is ion implantation. Previous work on implanted bismuth donors in silicon has demonstrated that the incorporation of ions using this technique is sufficient to fabricate high quality impurity qubit states. Such studies have produced bismuth spin qubits with an electron spin coherence time of $0.2 \mathrm{~ms}^{6}$ and a resolvable zero-field groundstate hyperfine doublet ${ }^{7}$. However, work still needs to be done in order to understand the fabrication techniques needed to produce a wide range of quantum devices. One example of this is the selection of the appropriate implantation dose parameters and understanding how this will translate to an active bismuth donor density. Implementing the appropriate donor density is of great importance when fabricating $\mathrm{Si}: \mathrm{Bi}$ samples that are to be used in the measurement of quantum phenomena. This is because it has been observed that the effects of concentration broadening plays a significant role in both the degradation of coherence times ${ }^{4}$ and reduction of available excited orbital transitions ${ }^{8}$.

This letter reports the effect that increasing the $\mathrm{Bi}^{+}$dose has on both the diffusion, and electrical activation yield of

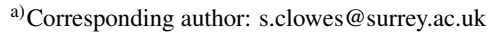

implanted bismuth donors in silicon. The characteristic properties of the $\mathrm{Si}: \mathrm{Bi}$ donor bound exciton, $D^{0} X$, complex is explored for each dose sample using PL measurements. These techniques are used in tandem to illustrate how the effects of bismuth diffusion can be observed in $D^{0} X$ luminescence and how the measured spectra can provide an indication of the quality of bismuth ion incorporation into the silicon lattice.

A $D^{0} X$ is formed when an electron and hole bind together through the Coulomb interaction to produce an exciton $(X$, the semiconductor equivalent of positronium) this exciton can then bind together with a neutral donor $\left(D^{0}\right)$ through the exchange interaction (analogous with positronium hydride) ${ }^{9}$. These systems can be considered as a single hole bound to a positively charged donor through a electron pair bond and have resonant formation energies of $1.149 \mathrm{eV}$ or $1.146 \mathrm{eV}$, in the case of phosphorus and bismuth donors respectively ${ }^{10}$. Once an exciton undergoes a recombination event, energy can be emitted optically leaving the donor in a neutral state ${ }^{11}$. Silicon is an indirect band gap medium which means that, during $D^{0} X$ recombination, the crystal momentum $(k)$ must be conserved. This leads to two possible mechanisms of PL which are often referred to as no-phonon (NP) and phonon assisted (PA) transitions. In a PA transition, $k$ is conserved by the generation of an accompanying lattice phonon. In this case, the energy of emitted radiation will be red-shifted from the NP transition by an amount equalling the phonon energy. NP recombination arises when the wave-function of the $D^{0} X$ has a very small spatial extent, i.e. the binding energy is large, so that its momentum uncertainty is large allowing $k$ conservation to be broken. The luminescence of both NP and PA $D^{0} X \mathrm{~s}$ are used in the analysis of samples in this study.

The silicon used in this implantation study was $\langle 111\rangle$ oriented float-zone with a bulk phosphorus impurity concentration of $(6.1 \pm 0.3) \times 10^{14} \mathrm{~cm}^{-3}$. Samples were fabricated us- 
ing a sequence of five bismuth implantation steps with cumulative ion fluences of $1.4 \times 10^{12} \mathrm{~cm}^{-2}, 4.5 \times 10^{12} \mathrm{~cm}^{-2}$, $1.4 \times 10^{13} \mathrm{~cm}^{-2}$ and $4.5 \times 10^{13} \mathrm{~cm}^{-2}$ and energies ranging from $400 \mathrm{keV}$ to $2000 \mathrm{keV}$. Samples have been labeled from A to $\mathrm{D}$ in order of increasing ion dose. A beam tilt angle of $7^{\circ}$ was use to inhibit ion channeling. These implant conditions were chosen to ensure that bismuth impurities would be deep enough to restrict undesirable $D^{0} X$ surface recombination channels and generate a PL signal that is large enough to be measurable. To repair crystal damage and incorporate bismuth atoms into lattice sites, substrates were annealed in a nitrogen atmosphere at a temperature of $900{ }^{\circ} \mathrm{C}$ for $300 \mathrm{~s}$ after implantation. This annealing treatment has previously been demonstrated to achieve bismuth ion incorporation in a crystal environment free from the effects of lattice strain ${ }^{7}$. Spreading resistance profiling, $\mathrm{SRP},{ }^{12}$ was used to evaluate the fraction of active bismuth donor sites. Samples were beveled at an angle of $0.005^{\circ}$ and the two-probe room temperature resistivity was measured down the angled face using a $5 \mathrm{mV}$ voltage source and an iterative step of $4 \mu \mathrm{m}$. In $D^{0} X$ PL measurements, above bandgap illumination was provided by a $532 \mathrm{~nm}$ laser which was incident on each of the implanted samples, mounted within a liquid helium cooled cryostat at a temperature of $10 \mathrm{~K}$. The photoexcitation laser was modulated using an optical beam chopper which, in conjunction with a lock-in amplifier, was used to filter out any signal from background noise. Emitted PL was analysed using a $1 \mathrm{~m}$ spectrometer using a slit width of $1 \mathrm{~mm}$ and a nitrogen cooled germanium detector with a diameter of $5 \mathrm{~mm}$.

SRP measurements (figures $1(\mathrm{a}-\mathrm{d})$ ) indicate that within every sample a population of n-type extrinsic doping has been introduced in the region predicted by Monte Carlo calculations (using the SRIM package ${ }^{13}$ ). For each sample it is clear that, in the depth region of 0.1 to $0.7 \mu \mathrm{m}$, the measured charge profiles are dominated by the implanted bismuth donors. However, as the depth from the surface of the substrate increases, the apparent bismuth donor population decreases and the charge concentration asymptotically approaches the known phosphorus density. Assuming an exponential decrease in bismuth donor population within the 'tail' region of the profile, and knowing the constant bulk density of phosphorus, a least squares model to fit to the measured charge profiles has been used to separate the contribution of each donor species. We define the integrated electrical activation yield (EAY-I) to be the total sheet concentration found from integrating the SRP over the depth, divided by the total implanted Bi fluence. Extracted EAY-I values (figure 1(e)) indicate that there is an inverse correlation between bismuth implantation dose and fractional activation. Substrates $C$ and D both achieve a EAY-I of approximately $60 \%$ of the implant dose which is consistent with other reports for similar density implants ${ }^{6}$. By comparison, in sample A, a donor yield of $(74 \pm 3) \%$ is achieved. While this is an encouragingly high EAY-I, it should be compared to the peak electrical activation yield (EAY-P) which is defined as the percentage of bismuth concentration at a given depth from SRP to that predicted by the SRIM calculation. In sample A the EAY-P is only $(57 \pm 3) \%$, and the fact that the EAY-P is lower than the

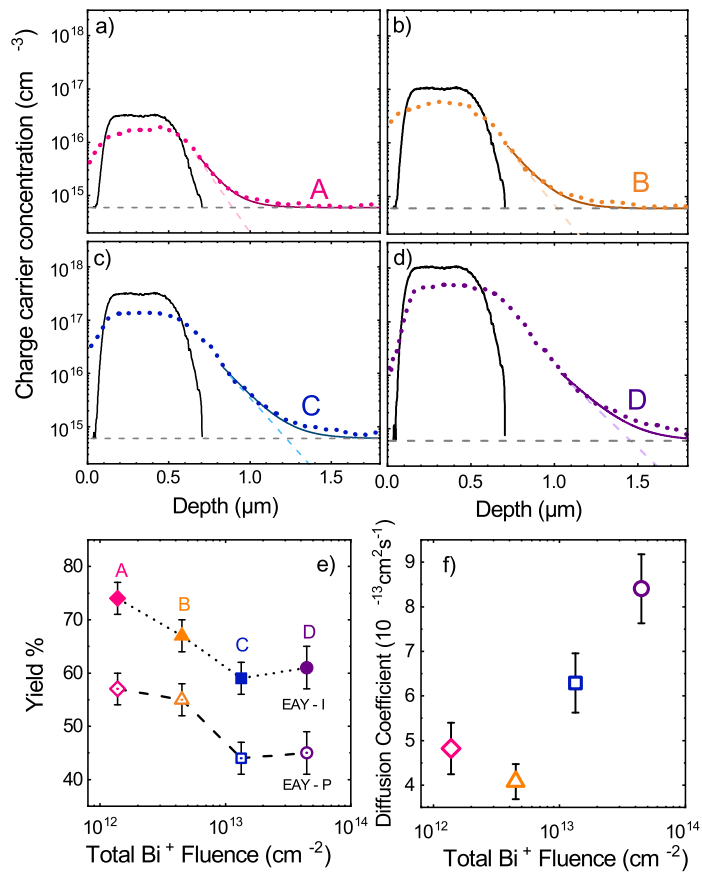

FIG. 1. $(a-d)$ Measured spreading resistance profile donor depth distributions for each of the samples A to D shown by coloured dots. For each sample, the solid black line shows a Monte Carlo simulation for the implant depth profile before annealing/activation. Dashed grey lines indicate the bulk concentration of phosphorus donors preexisting in the substrate before the implant whereas coloured dashed lines are diffused bismuth donors which are extracted by fitting to the SRP data (solid coloured lines). (e) Measured percentage integrated yield (EAY-I) and peak yield (EAY-P) for each of the four density samples (as indicated). (f) Extracted diffusion coefficients for each donor profile.

EAY-I indicates that diffusion of bismuth ions has occurred. This effect is observable in each of samples in this study where the EAY-P is consistently $\sim 15 \%$ less than the EAY-I would suggest.

By evaluating the shape of the measured SRP profiles, the significance of diffusion becomes clearer. The energies of each implantation step are consistent between samples and it is to be expected that the shape of each bismuth ion stopping distribution would be identical. This is reflected in the calculated SRIM distributions. However, as the implant fluence increases, the active donor concentration profile penetrates deeper into the substrate. Using the simulated SRIM distributions as a starting point, and knowing the temperature and duration of the annealing treatment, it is possible to extract a $\mathrm{Bi}^{+}$diffusion coefficient for each of the measured SRP distributions. Calculated diffusion coefficients are displayed in figure 1(f). There is a clear correlation between the $\mathrm{Bi}^{+}$ implantation fluence and the magnitude of bismuth migration. This therefore indicates that the nature of diffusion observed in these substrates is inherent to the damage caused by the implantation. Furthermore, the extracted diffusion coefficients 
are much larger than would be expected if the bismuth impurity movement were limited solely to thermal diffusion or interactions with an equilibrium population of vacancies. At a temperature of $900{ }^{\circ} \mathrm{C}$, the equilibrium bismuth diffusivity is is estimated to be $3.6 \times 10^{-15} \mathrm{~cm}^{2} \mathrm{~s}^{-1}$ from extrapolating the Arrhenius relation as described by Ishikawa et $\mathrm{al}^{14}$. By comparison the extracted diffusion coefficients from this study are of a magnitude that would be expected for annealing temperatures above $120^{\circ} \mathrm{C}$.

During the $\mathrm{Bi}^{+}$implant, traveling ions create an abundance of silicon crystal vacancies and interstitials through collisions with lattice sites. These point defects are known to be extremely mobile and, during annealing, coalesce to create extended defects ${ }^{15}$. It is well established that the presence of lattice damage can significantly enhance the migration of implanted impurity states. One key example of this is known as transient enhanced diffusion, TED, which is most often in associated with the diffusion of phosphorus and boron atoms which pair with $\mathrm{Si}$ interstitials ${ }^{16,17}$. Bismuth on the other hand is known to favour pairing with silicon lattice vacancies to accommodate diffusion due to its large covalent radius and much lower activation energy ${ }^{14}$. It is therefore reasonable to suggest that the abundance of lattice vacancies created by the increasing dose of implanted $\mathrm{Bi}$ ions directly contributes to the enhanced diffusion observed in this study.

To evaluate the characteristic properties of active bismuth donors, $D^{0} X$ PL measurements were taken using each of the samples in this study. Figure 2(a) displays a reference PL measurement of un-implanted silicon. Included is an identification of the spectral regions within which the $D^{0} X$ transverse optical phonon, TO, and NP transitions are expected (b $\&$ c respectively). These are situated either side of the significantly stronger transverse optical phonon mediated silicon bandgap PL, Si BG(TO). Figures 2(b \& c) display measured spectra of the implanted samples in this study, peaks centred at $1147 \mathrm{meV}$ and $1150 \mathrm{meV}$ correspond to the NP $D^{0} X$ PL for bismuth and phosphorus donors respectively. Transitions at $1137 \mathrm{meV}$ correspond to the recombination of free excitons assisted by the creation of a transverse acoustic phonon, $\mathrm{Si}$ BG(TA). Figure 2(d) displays the integrated spectral density of both Si:Bi $D^{0} X$ PL centres as a function of active bismuth donor population. For samples A to $\mathrm{C}$, the NP and TO spectral intensities scale lineally with donor population and it is found that the TO:NP transition intensity ratio match the known Si:Bi $D^{0} X$ PL branching ratio $(1: 2.2)^{10}$. This serves as an indication that the crystal environment, within which the bismuth donors are populated, is of relatively high quality. However in sample D the PL strength is found to be greatly reduced from what would be extrapolated from the lower density substrates. Accompanying this reduction in the expected $D^{0} X$ PL centre from sample $D$ is the emergence of a lower energy feature present in the $D^{0} X$ (TO) spectrum at $(1083.57 \pm 0.09) \mathrm{meV}$. It is not clear if this feature is also present in the equivalent $\mathrm{NP}$ measurement due to the overlapping position of the $\mathrm{Si}$ $\mathrm{BG}(\mathrm{TA})$ transition. It is also of note that, at the expected luminescence energy, the TO:NP intensity ratio from sample D does match the known 1:2.2 branching ratio.

An identification for the shifted low energy peak can be pro-
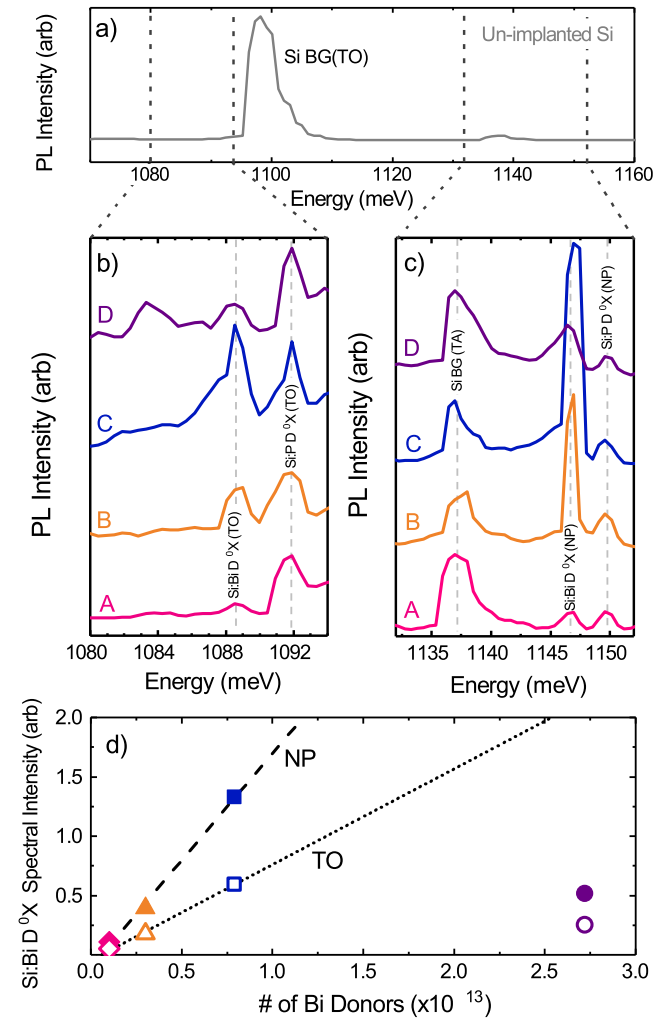

FIG. 2. (a) Reference PL spectra of un-implanted silicon. $(b-c)$ PL spectra of samples A to D. NP and TO phonon assisted $D^{0} X$ transition energies for both bismuth and phosphorus donors are indicated as dashed grey lines. (d) Integrated $\mathrm{Si}: \mathrm{Bi} D^{0} X$ spectral density as a function of total number of active Bi donors for each of the four samples. Linear fits to the PL of samples A to $\mathrm{C}$ are included as dashed and dotted lines for NP and TO centres respectively.

vided by considering the formation of the $D^{0} X$ complex in an environment with a dense population of bismuth donors. The interaction between excitons and multiple donors has been reported previously in the cases of both bulk and ion implanted Si:P substrates. The energy of $D^{0} X$ PL has been observed to shift down in energy by as much as $20 \mathrm{meV}$ with increasing concentrations ${ }^{18,19}$. Much like a pair of hydrogen atoms, neighbouring pairs of group $\mathrm{V}$ donors in silicon are known to form bonding states with reduced overall energy through the exchange interaction ${ }^{20}$. Similarly, if a bound exciton is shared between two $D^{0}$ (analogous to positronium di-hydride), there can be an overall reduction in the corresponding $D_{2}^{0} X$ binding energy. By approximating the wavefunction of the $D^{0} X$ complex as a sphere with a radius of $r_{0}$, the binding energy of an exciton bound to a pair of neutral donors with separation $R$ $\left(>r_{0}\right)$ is ${ }^{21}$

$$
E_{2}=E_{1}\left[1+2 \exp \left(-R / r_{0}\right)\right]
$$

where $E_{1}$ is the energy required to dissociate a bound exciton from a single donor, $8.8 \mathrm{meV}$ in the case of $\mathrm{Si}: \mathrm{Bi}^{10}$. The $D^{0} X$ 


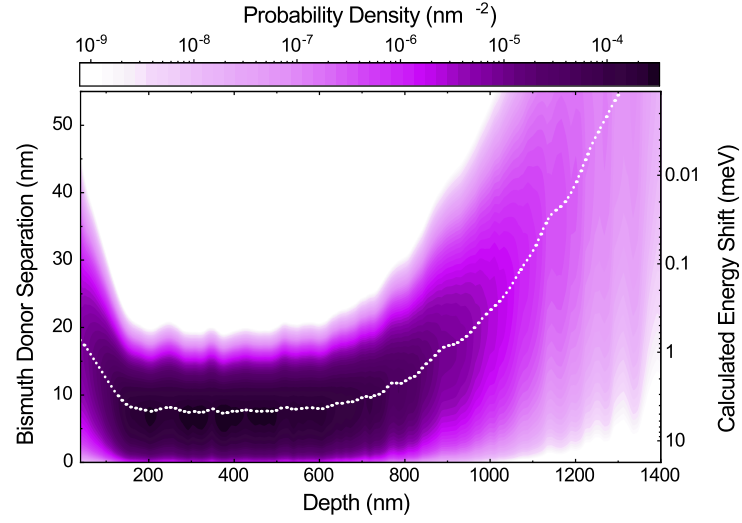

FIG. 3. Probability density distribution of bismuth donor separations (and predicted energy shift $\left|E_{2}-E_{1}\right|$, Eqn 1, for the bi-donor bound mono-exciton $D_{2}^{0} X$ relative to the $\left.D^{0} X\right)$ as a function of depth for implant $\mathrm{D}$. The dotted line contours the expectation value of separations at each depth.

radius, $r_{0}$, may be approximated by the sum of the free exciton and donor Bohr radii, $4.9 \mathrm{~nm}^{22}$ and $1.63 \mathrm{~nm}^{23}$ respectively. Clearly, as the spacing between donors decreases, the energy shift caused by this clustering effect increases as expeced.

Figure 3 displays, for sample $\mathrm{D}$, the probability of finding a bismuth donor that is within a depth range $z \rightarrow z+d z$ below the surface, and that has its nearest neighbour within the radial distance range $R \rightarrow R+d R$. This probability density assumes complete spatial randomness within the plane perpendicular to the ion beam, and a non-homogeneous Poisson point process with its density variation in depth found from the measured SRP density data (figure $1(\mathrm{a}-\mathrm{d}))^{24}$. The expectation nearest neighbour radius was then calculated from this probability density function and is plotted as the white dotted line in figure 3. As to be expected, the nearest neighbour donor separation reaches a minimum within the high density 'flat top' region of the sample at depths ranging from 0.1 to $0.7 \mathrm{~nm}$ below the surface of the sample. Within this population the donor separation expectation value is $(7.6 \pm 0.2) \mathrm{nm}$ which corresponds to a calculated energy shift of $-(4.6 \pm$ $0.4) \mathrm{meV}$. This predicted value is in good agreement with the $-(4.8 \pm 0.1) \mathrm{meV}$ shift measured experimentally in the Si:Bi $D^{0} X$ (TO) PL spectra.

There is also significance in the fact that, within the PL measured from sample $D$, there remains a spectral feature present at the expected Si:Bi $D^{0} X$ energy that exhibits the expected 1:2.2 TO:NP branching ratio. This can be identified as a population of bismuth donors which are not subjected to any concentration effects. Using the linear spectral intensity relations displayed for the lower density samples (figure 2(d)), this PL strength can be correlated to a donor population of approximately $(3.3 \pm 0.2) \times 10^{13}$. This number matches well the population of bismuth donors which are measured to be at depths greater than $900 \mathrm{~nm}$ below the surface. As donors diffuse to greater depths, the population density drops significantly and the resultant nearest neighbour separation increases correspondingly. Thus, $D^{0} X$ PL will not undergo any measurable density effects.

The effect of increasing the ion implantation dose on the characteristic properties of implanted bismuth donors in silicon has been studied. We found that implants with a lower ion fluence produced higher EAY-I, the total (integrated) electrical activation density when measured as a fraction of the total fluence. The peak in the activation density as a fraction of the simulated peak implant density EAY-P, was observed to be significantly less than the total yield in every sample, and correspondingly the thickness of the annealed layer was larger than the implant profile. A clear correlation was observed between an increasing $\mathrm{Bi}^{+}$dose and faster bismuth diffusion. This is consistent with diffusion of bismuth ions through the silicon crystal during the anneal via interactions with silicon vacancies and interstitials. PL measurements showed, for every sample, features corresponding to both the implanted bismuth and bulk doped phosphorus donors. For the highest density sample, we observed a suppression in the $D^{0} X$ transition from isolated $\mathrm{Si}: \mathrm{Bi}$ donors, and an additional low energy peak was also measured. We have identified the latter as due to excitons interacting more loosely with a pair of bismuth donors. The spectral feature associated with isolated $\mathrm{Si}: \mathrm{Bi}$ donors is not suppressed completely, thanks to significant numbers of donors situated within the diffused tail where densities are correspondingly lower. These results demonstrate the significance in careful selection of appropriate implantation doses when considering the fabrication of quantum devices centered around active bismuth impurity states in silicon.

We gratefully acknowledge the financial support from the UK Engineering and Physical Sciences Research Council [ADDRFSS, Grant No.EP/M009564/1].

${ }^{1}$ J. P. Gordon and K. D. Bowers, Phys. Rev. Lett. 1, 368 (1958).

${ }^{2}$ G. Feher, Phys. Rev. 114, 1219 (1959).

${ }^{3}$ K. Saeedi, M. Szech, P. Dluhy, J. Z. Salvail, K. J. Morse, H. Riemann, N. V. Abrosimov, N. Nötzel, K. L. Litvinenko, B. N. Murdin, et al., Scientific Reports 5, 10493 (2015).

${ }^{4}$ G. Wolfowicz, A. M. Tyryshkin, R. E. George, H. Riemann, N. V. Abrosimov, P. Becker, H.-J. Pohl, M. L. W. Thewalt, S. A. Lyon, and J. J. L. Morton, Nature Nanotechnology 8, 881 (2013), 1301.6567.

${ }^{5}$ T. Sekiguchi, M. Steger, K. Saeedi, M. L. W. Thewalt, H. Riemann, N. V. Abrosimov, and N. Nötzel, Physical Review Letters 104 (2010).

${ }^{6}$ C. D. Weis, C. C. Lo, V. Lang, A. M. Tyryshkin, R. E. George, K. M. Yu, J. Bokor, S. A. Lyon, J. J. L. Morton, and T. Schenkel, APL 100, 172104 (2012).

${ }^{7}$ T. Peach, K. Homewood, M. Lourenco, M. Hughes, K. Saeedi, N. Stavrias, J. Li, S. Chick, B. Murdin, and S. Clowes, Advanced Quantum Technologies 1, 1800038 (2018).

${ }^{8}$ G. a. Thomas, M. Capizzi, F. DeRosa, R. N. Bhatt, and T. M. Rice, Phys. Rev. B 28, 5472 (1981).

${ }^{9}$ P. J. Dean, J. R. Haynes, and W. F. Flood, Phys Rev 161, 711 (1967).

${ }^{10}$ J. R. Haynes, Physical Review Letters 4, 361 (1960).

${ }^{11}$ G. Davies, Physics reports 176, 83 (1989).

${ }^{12}$ R. Brennan and D. Dickey, Solecon Labs Technical Note.

${ }^{13}$ J. Ziegler, M. Ziegler, and J. Biersack, Nuclear Inst. and Methods in Physics Research, B 268, 1818 (2010).

${ }^{14}$ Y. Ishikawa, K. Yazaki, and I. Nakamichi, Jpn. J. Appl. Phys. 28, 1272 (1989).

${ }^{15}$ A. Claverie, B. Colombeau, B. de Mauduit, C. Bonafos, X. Hebras, G. Ben Assayag, and F. Cristiano, Appl Phys A 76, 10251-(2003).

${ }^{16}$ K. Cho, M. Numan, T. Finstad, and W. K. Chu, APL 47, 1321 (1985).

${ }^{17}$ K. Jones, H. Banisaukas, and J. Glassberg, APL 75, 3659 (1999).

${ }^{18}$ Y. Shiraki and H. Nakashima, Solid State Comms 29, 295 (1979). 
${ }^{19}$ H. Sumikura, K. Nishiguchi, Y. Ono, A. Fujiwara, and M. Notomi, Optics Express 19, 25255 (2011).

${ }^{20}$ A. Miller and E. Abrahams, Phys. Rev. 120, 745 (1960).

${ }^{21}$ K. Narita, Solid State Communications 29, 299 (1979).

${ }^{22} \mathrm{P}$. Yu and M. Cardona, Fundamentals of Semiconductors: Physics and Ma- terials Properties (Springer-Verlag Berlin Heidelberg, 2010).

${ }^{23}$ J. Li, N. H. Le, K. L. Litvinenko, S. K. Clowes, H. Engelkamp, S. G. Pavlov, H.-W. Hübers, V. B. Shuman, L. Portsel, A. N. Lodygin, et al., Phys. Rev. B 98, 085423 (2018).

${ }^{24}$ K. Stockbridge, S. Chick, E. Crane, A. Fisher, and B. Murdin, (2019), Under peer review. 

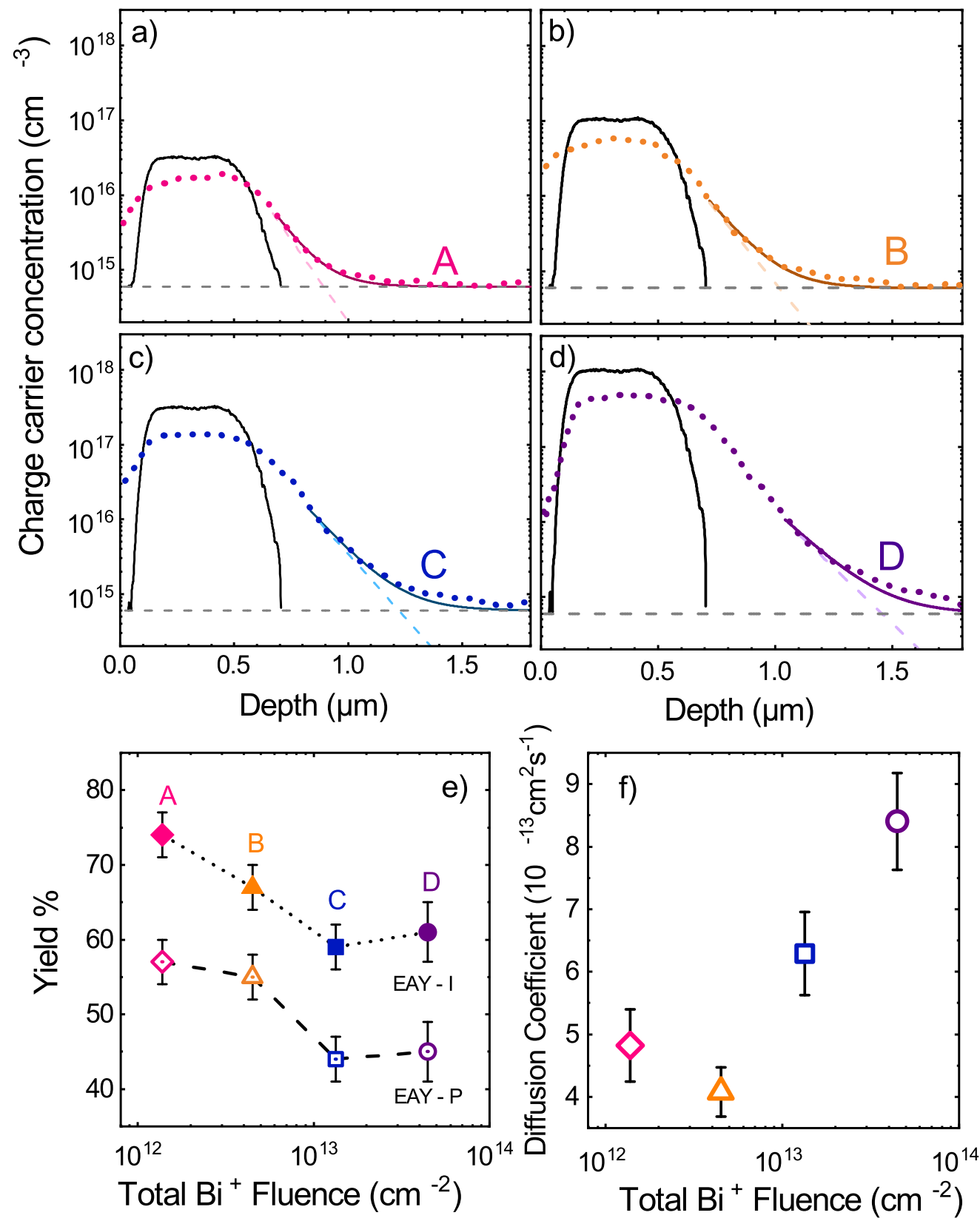

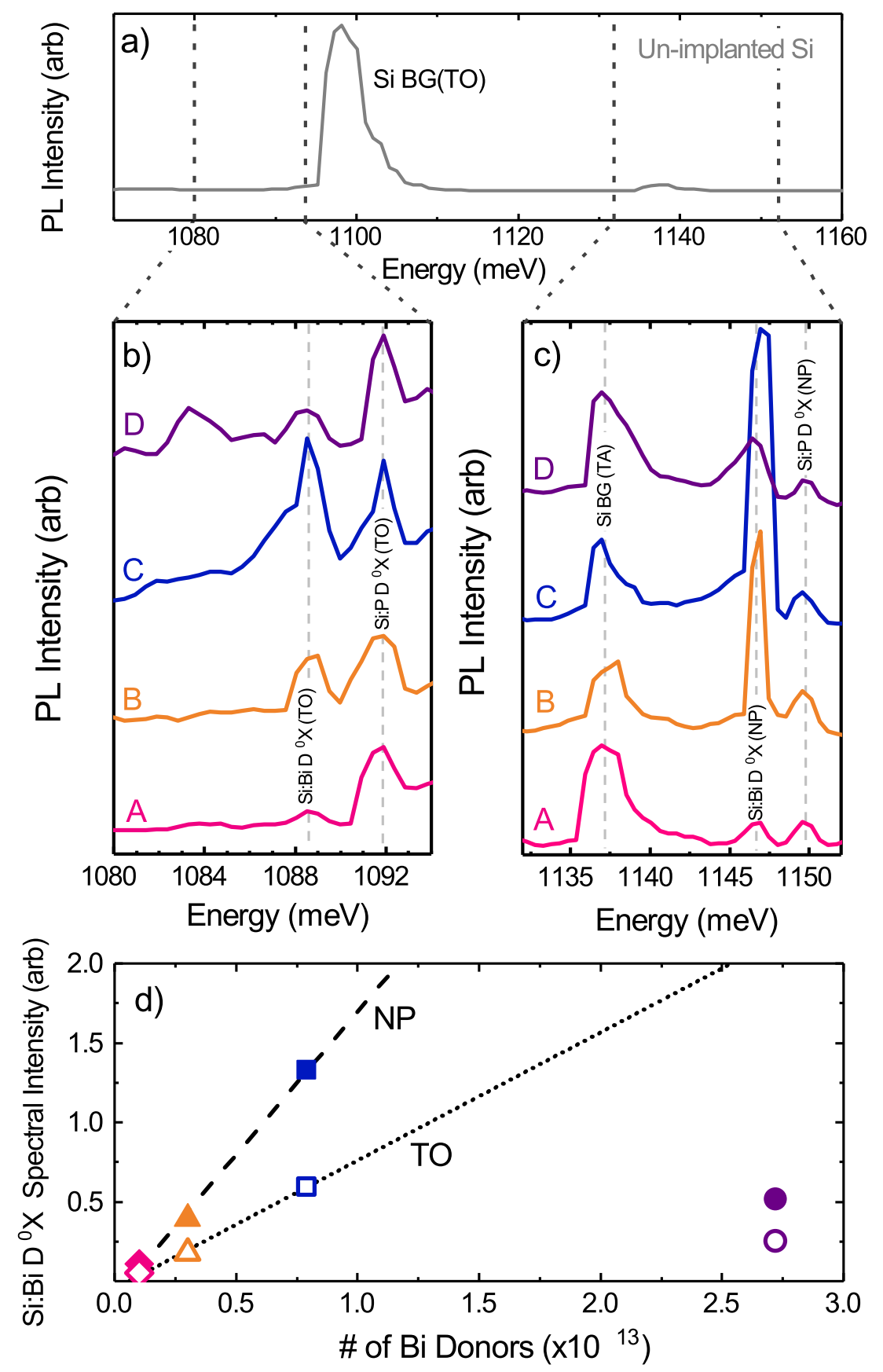


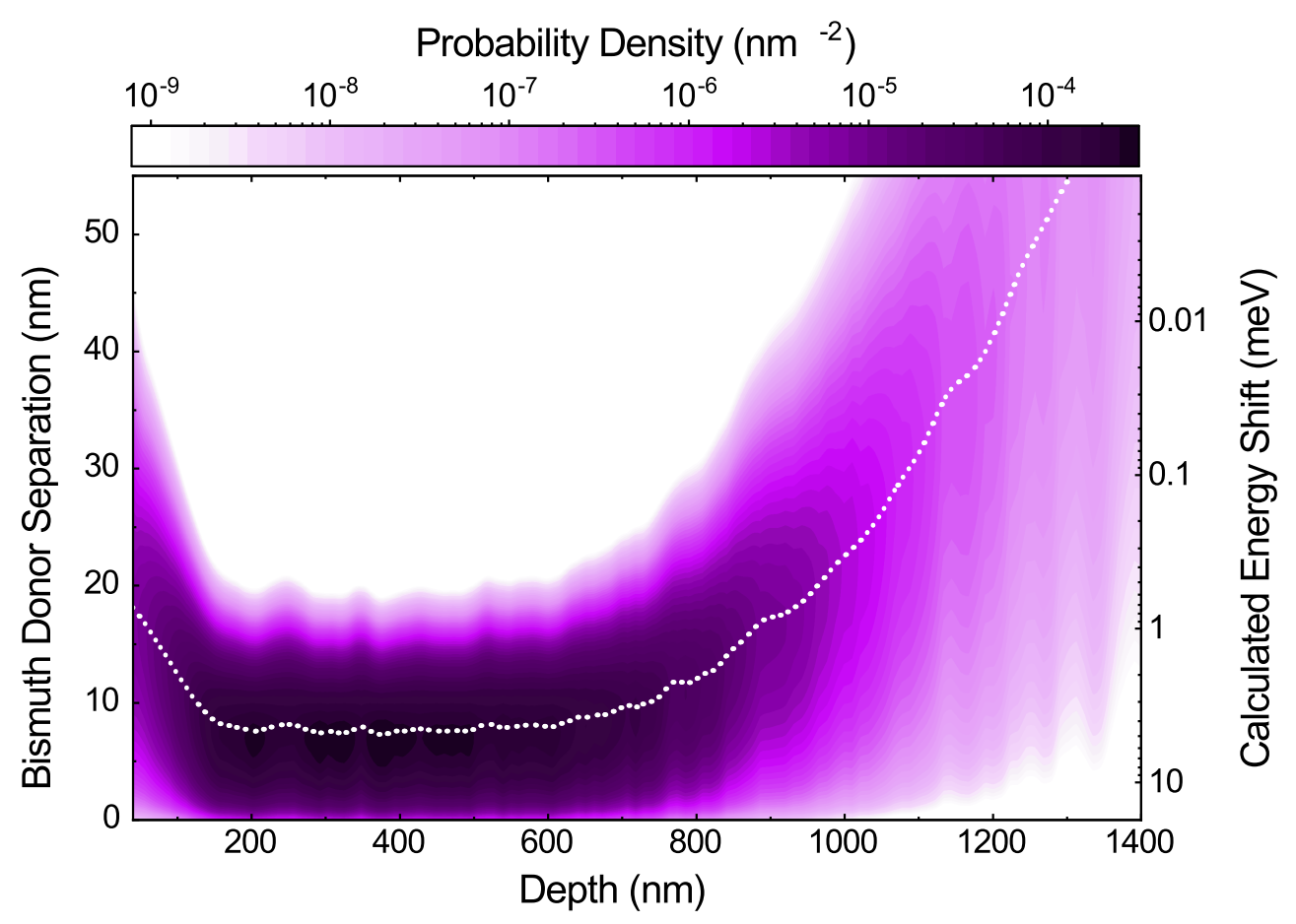

